\title{
First Report of Geniospasm in a Five-Generation Brazilian Family: Clinical Features, Electromyography Findings and Botulinum Tox- in Treatment
}

\author{
Carvalho LB*, Rampim DB and Chung TM \\ Institute of Physical Medicine and Rehabilitation, Hospital das Clínicas, University of São Paulo, School of Medicine, Brazil
}

*Corresponding author: Lilian Braighi Carvalho, MD, Institute of Physical Medicine and Rehabilitation, Hospital das Clínicas, University of São Paulo, School of Medicine, Brazil, IMREA-HC/USP, Ovídio Pires de Campos Street, 300 Gate 3, Jardim Paulista, São Paulo - SP, Brazil, Zip Code 05403-000, E-mail: lilian.braighi@hc.fm.usp.br

\begin{abstract}
Geniospasm is a rare movement disorder characterized by spontaneous, involuntary, irregular trembling of the chin and the lower lip with accompanying involuntary contractions of the mentalis muscle. It is thought to be an autosomal-dominant inherited condition with high penetrance, which typically manifests in early life and is triggered or exacerbated by stress. When present in adults, the paroxysmal, rhythmic up-and-down movements of the lower face can lead to distress, social embarrassment and sleep impairment. It must be differentiated from other conditions, such as tic, facial myokymia and jaw tremor. In this paper, we report the first cases of geniospasm in a five-generation Brazilian family. We describe its genealogy and clinical features, the findings of electromyography testing, and the successful treatment of four living members of three generations using botulinum toxin injections. A further study amongst surviving members of the family is planned in order to evaluate genetic mutations underlying geniospasm by genomic analysis using exome sequencing.
\end{abstract}

\section{Keywords}

Geniospasm, Hereditary chin trembling, Autosomal-dominant movement disorder, Chin myoclonus, Chin quivering

\section{Introduction}

Geniospasm (hereditary chin trembling) is an unusual movement disorder characterized by spontaneous, intermittent, involuntary trembling of the chin and, in rare cases, the lower lip. It was first described in the medical literature in 1894 by Domenico Massaro, who reported 26 cases in the same family in Italy over five generations [1]. In 1922, Percy Stocks reported geniospasm for the first time in a British family [2]. He described the condition as an inherited facial spasm confined to the mentalis muscles, distinguishing it from facial tics [2]. Geniospasm is also distinguished from tremor, as it is not necessarily rhythmic, and unlike tremor, can be seen during sleep $[3,4]$. Rather, it should be classified as hereditary chin myoclonus [5]. Episodes of geniospasm last from seconds to hours and can be triggered or intensified by anxiety, emotion, concentration, anger or stress [6]. The condition, which is considered benign, typically manifests in early life, with episodes tending to reduce in frequency/intensity with advancing age [7]. If present in adults, it can lead to distress, social embarrassment and sleep impairment $[7,8]$; in these cases, treatment with botulinum toxin injections should be considered as an effective and well-tolerated approach [9-11].

Geniospasm is thought to be an autosomal-dominant inherited condition, which displays genetic heterogeneity [8]. From a search of the international medical literature, we found reports of geniospasm in 28 families, mostly from Europe and North America [1-3,5,6,830]. There have been four reports in families in Asia $[11,26-28]$ and two in South America $[29,30]$. In this paper, we present the first report of geniospasm across five generations of a Brazilian family. We describe its clinical features, the use of Electromyography (EMG) testing and the effectiveness of treatment with botulinum toxin injections. 


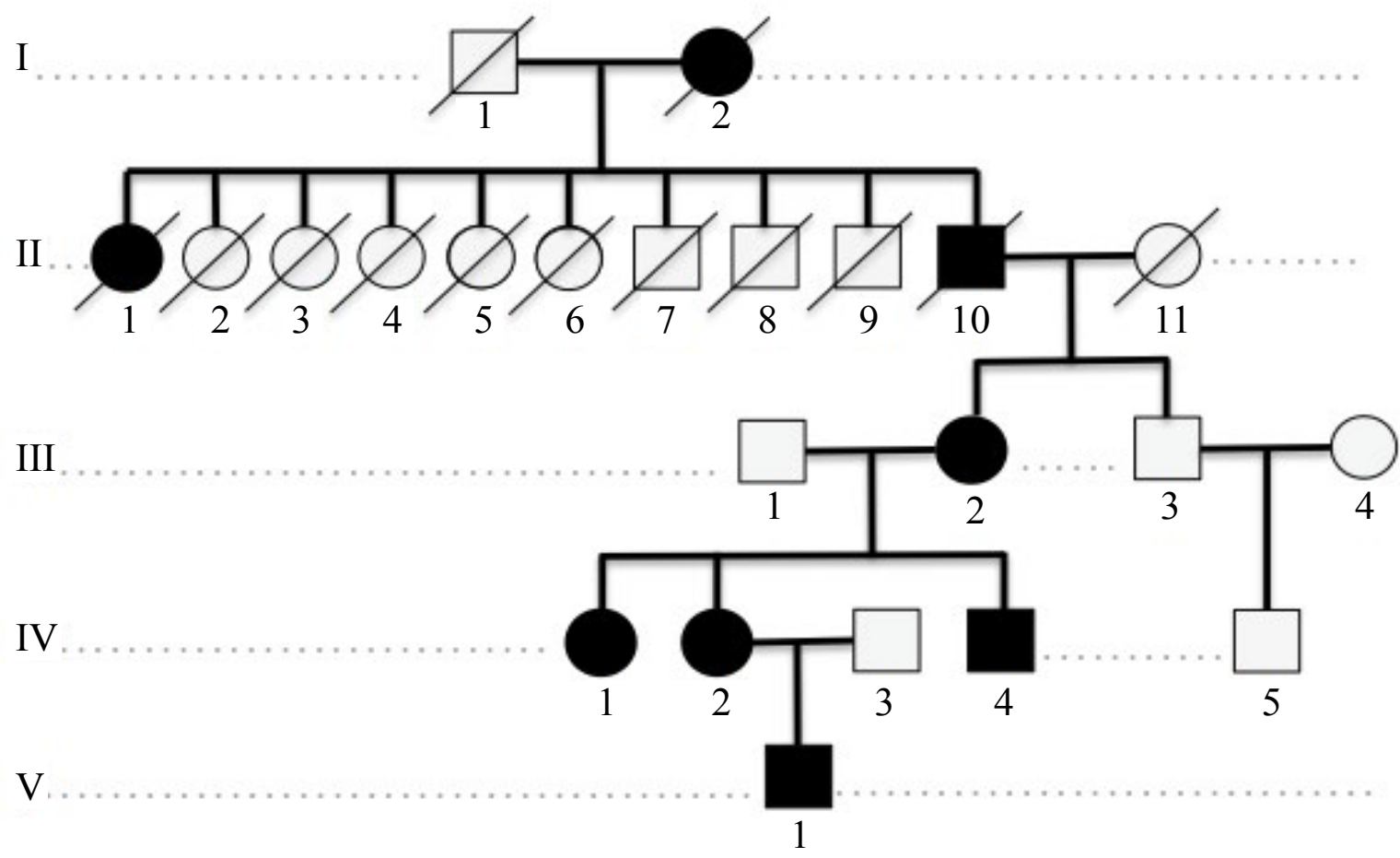

Figure 1: Family tree of Brazilian family members affected by geniospasm.

Affected family members are shown in black; square represent males and circles represent females; diagonal lines represent deceased family members.

\section{Clinical Features}

Four members of a Brazilian family from São Paulo (a mother, two daughters and a son) first reported to our clinic (Neuromuscular Blockade Ambulatory, Institute of Physical Medicine and Rehabilitation, Hospital das Clinicas, University of São Paulo School of Medicine IMREA-HC/USP) with symptoms of chin trembling on 5 October 2007. The family were Caucasian and of Portuguese descendance. One of the daughters subsequently gave birth to a boy who also manifested the symptoms of geniospasm in the early months of life. A review of the family tree revealed three other family members with similar symptoms (Figure 1). None of the family members have any other clinical disease or neurological impairment.

Symptoms appeared to be most severe in the elder daughter (IV-1), who had periodic involuntary activity of the mentalis muscles. The duration was unpredictable, but chin trembling could be observed many times a day. The intermittent movements started at the mentalis muscle, spreading to her lips and to an area close to her nose. As well as the mentalis muscle, the orbicularis oris and mylohyoid muscles were also affected, with partial involvement of the levator labii superioris muscle. The patient complained that the intermittent involuntary movement in her lower face caused irritation during the day and led to bruxism during the night, and consequently sleep impairment.

The son (IV-4) had clinical evidence of trembling of the mentalis and orbicularis oris muscles; on rare occasions, the mylohyoid muscle was also involved. As a result, he had serious problems with bruxism and clinical evidence of masseter muscle hypertonia, leading to impairments in speech and chewing. All of these symptoms were observed during the first few months of his life and, although he was not the most affected member of the family, he found his symptoms extremely disabling in a social context.

The mother (III-2) and the second daughter (IV-2) were less affected. The mother had trembling of the mentalis muscles and, less frequently, of the orbicularis oris muscle and depressor anguli oris muscle. The daughter was the only family member who had trembling confined to mentalis muscles. Neither the mother nor the second daughter had symptoms of bruxism. The second daughter had a son (V-1) in 2012. He manifested chin trembling during the first months of life, especially when playing with toys that required attention and concentration.

Investigation of the family tree revealed that three other members also had symptoms of geniospasm. The great-grandmother (I-2) had chin trembling, but was not investigated further. The grandfather (II-10) had the same symptoms, which could be observed during soccer matches. His sister (II-1) also had the same symptoms, but died at a very young age and did not have children.

\section{Electromyography testing}

EMG tests were conducted on the affected muscles of the four symptomatic adults (III-2, IV-1, IV-2 and IV-4). This involved placement of a mono polar needle in the target muscle while patients were lying on a bed with 


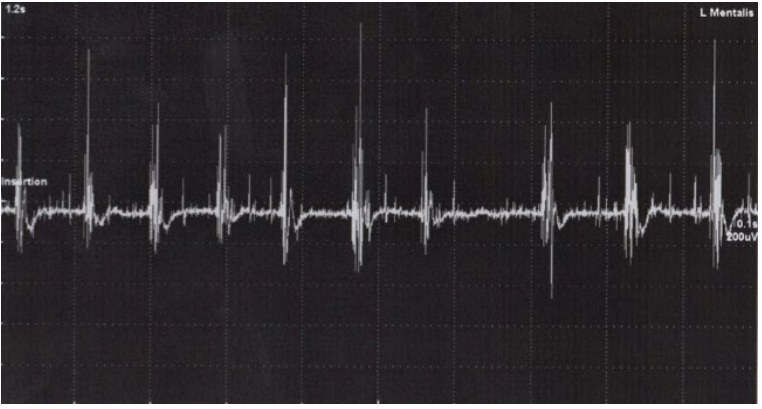

A. Left mentalis, patient III-2.

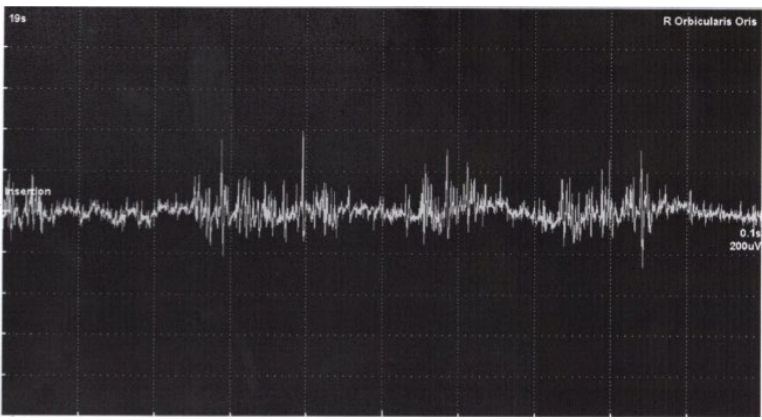

C. Right orbicularis oris, patient IV-4.

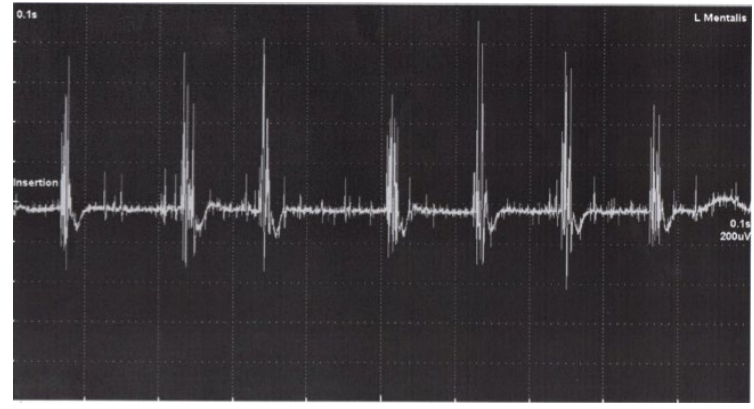

B. Left mentalis, patient IV-2.

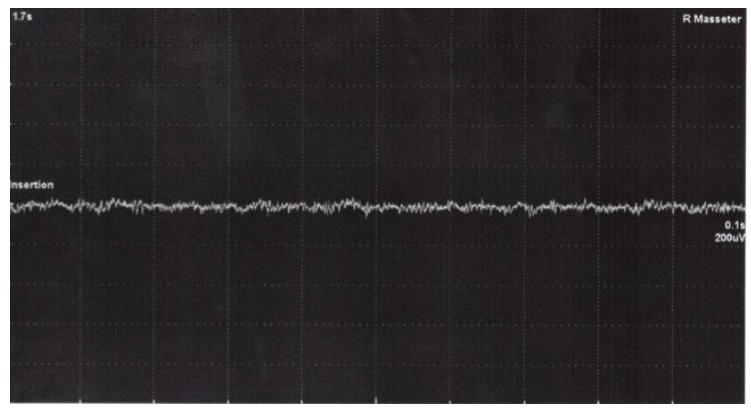

D. Right masseter, patient IV-4.

Figure 2: Involuntary muscle activity in mentalis and orbicularis oris muscles in Brazilian family members with geniospasm.

their eyes closed. Involuntary muscle activity was then recorded using a Nihon Kohden machine (four-channel EMG/EP system).

Locating target muscles using EMG is straightforward. The needle tip is positioned in such a way that a full recruitment pattern can be seen with activation of the targeted muscle. Motor unit action potential should have normal amplitude and short rise times, making a distinctly crisp sound [31]. The presence of a crisp sound only indicates that the needle tip is placed near a contracting fascicle; it does not necessarily guarantee proper placement in the targeted muscle. Confirmation of the correct location is therefore required by active contraction or passive movement of the targeted muscle, while checking the EMG signals or the needle movement, respectively. In our clinic, we use a sensitivity of $0.2 \mathrm{mV}$ per division and sweep speed of $10 \mathrm{~ms}$ per division to record muscle activation.

Results of EMG testing of the mentalis and other affected muscles showed involuntary activity and motor units of normal morphology firing at $6-10 \mathrm{~Hz}$. Figure 2 shows sample EMG traces of the mentalis, orbicularis oris and masseter muscles of affected family members. In the mentalis muscles, a tremulous pattern was noted, characterized by rhythmic bursts, generally lasting between 50 and 300 ms. In the orbicularis oris muscles, an irregular phasic pattern was seen, with bursts of variable amplitude, rhythmicity and duration lasting $300 \mathrm{~ms}$ to several seconds. In the patients reporting bruxism, there was no involuntary activity in the masseter muscle.

\section{Botulinum toxin treatment}

The decision to initiate botulinum toxin treatment in patients III-2, IV-1, IV-2 and IV-4 was discussed with all family members and made on the basis of uncomfortable trembling and social impairment. Between November 2007 and 2015, these patients have each received a total of 14 botulinum toxin injections administered every 5-9 months, except for patient IV-2, who received 13 (one injection missed owing to pregnancy). The muscles injected and dosages chosen were predominantly based on clinical evaluation of the affected muscles alongside EMG findings and the clinical experience of the injectors. For their first injections, patients III-2, IV-1 and IV-4 received 15 units of botulinum toxin (Dysport ${ }^{\circledR}$ ) into each mentalis muscle, while patient IV-2 received 10 units into each mentalis muscle. For subsequent injections, the dosage was increased to 25-30 units for each mentalis muscle; other associated muscles were also injected: Orbicularis oris (4 units), mylohyoid (40 units) and levator labii superioris (6 units). The dosages and schedule used are shown in Table 1.

For patients IV-1 and IV-4, the decision was made to also inject the masseter muscles to treat the bruxism symptoms. At the fifth injection, patient IV-4 received 80 units of botulinum toxin into the masseter muscles on each side. At the sixth injection, patient IV-1 received 50 units into the masseter muscles.

At the tenth injection, the botulinum toxin formulation was changed from Dysport ${ }^{\circledR}$ to Botox ${ }^{\circledR}$. The decision to change was discussed with all family members and the rationale for this change was to extend the effects of botulinum toxin neuromuscular blockade. The dosage was: Mentalis muscles (10-15 units each), orbicularis oris muscle (3 units each), mylohyoid (30 units), 
Table 1: Dosage schedule.

\begin{tabular}{|c|c|c|c|c|c|c|}
\hline Injection & Date & Botulinum toxin & Patient III-2 & Patient IV-1 & Patient IV-2 & Patient IV-4 \\
\hline 1 & $23 / 11 / 2007$ & Dysport $^{\circledR}$ & $\begin{array}{l}\text { Mentalis } 15 \mathrm{U} \text { each } \\
\text { side (30U) } \\
\text { Total: } 30 \mathrm{U}\end{array}$ & $\begin{array}{l}\text { Mentalis } 15 \mathrm{U} \text { each side } \\
(30 \mathrm{U}) \\
\text { Total: } 30 \mathrm{U}\end{array}$ & $\begin{array}{l}\text { Mentalis } 10 U \text { each } \\
\text { side }(20 U) \\
\text { Total: } 20 U\end{array}$ & $\begin{array}{l}\text { Mentalis } 15 U \text { each } \\
\text { side (30U) } \\
\text { Total: } 30 U\end{array}$ \\
\hline 2 & $27 / 06 / 2008$ & Dysport $^{\circledR}$ & $\begin{array}{l}\text { Mentalis } 30 \mathrm{U} \text { each } \\
\text { side }(60 \mathrm{U}) \\
\text { Total: } 60 \mathrm{U}\end{array}$ & $\begin{array}{l}\text { Mentalis } 30 \mathrm{U} \text { each side } \\
(60 \mathrm{U}) \\
\text { Total: } 60 \mathrm{U}\end{array}$ & $\begin{array}{l}\text { Mentalis } 20 U \text { each } \\
\text { side }(40 U) \\
\text { Total: } 40 U\end{array}$ & $\begin{array}{l}\text { Mentalis } 15 U \text { each } \\
\text { side (30U) } \\
\text { Total: } 30 U\end{array}$ \\
\hline 3 & $16 / 01 / 2009$ & Dysport $^{\circledR}$ & $\begin{array}{l}\text { Mentalis } 25 \mathrm{U} \text { each } \\
\text { side (50U) } \\
\text { Total: } 50 \mathrm{U}\end{array}$ & $\begin{array}{l}\text { Mentalis } 25 \mathrm{U} \text { each side } \\
(50 \mathrm{U}) \\
\text { Total: } 50 \mathrm{U}\end{array}$ & $\begin{array}{l}\text { Mentalis } 15 U \text { each } \\
\text { side (30U) } \\
\text { Total: } 30 U\end{array}$ & $\begin{array}{l}\text { Mentalis } 20 U \text { each } \\
\text { side }(40 U) \\
\text { Total: } 40 U\end{array}$ \\
\hline 4 & $30 / 10 / 2009$ & Dysport $^{\circledR}$ & $\begin{array}{l}\text { Mentalis } 15 U \text { each } \\
\text { side (30U) } \\
\text { Total: } 30 U\end{array}$ & $\begin{array}{l}\text { Mentalis } 30 \mathrm{U} \text { each side } \\
(60 \mathrm{U}) \\
\text { Total: } 60 \mathrm{U}\end{array}$ & $\begin{array}{l}\text { Mentalis } 15 U \text { each } \\
\text { side }(30 U) \\
\text { Total: } 30 U\end{array}$ & $\begin{array}{l}\text { Mentalis } 20 U \text { each } \\
\text { side (40U) } \\
\text { Total: } 40 U\end{array}$ \\
\hline 5 & $25 / 06 / 2010$ & Dysport $^{\circledR}$ & $\begin{array}{l}\text { Mentalis } 15 U \text { each } \\
\text { side (30U) } \\
\text { Total: } 30 U\end{array}$ & $\begin{array}{l}\text { Mentalis } 30 \mathrm{U} \text { each side } \\
(60 \mathrm{U}) \\
\text { Mylohyoid } 20 \mathrm{U} \text { each } \\
\text { side }(40 \mathrm{U}) \\
\text { Total: } 100 \mathrm{U}\end{array}$ & $\begin{array}{l}\text { Mentalis } 15 U \text { each } \\
\text { side (30U) } \\
\text { Total: } 30 U\end{array}$ & $\begin{array}{l}\text { Mentalis } 20 \mathrm{U} \text { each } \\
\text { side }(40 \mathrm{U}) \\
\text { Masseter } 80 \mathrm{U} \text { each } \\
\text { side }(160 \mathrm{U}) \\
\text { Total: } 200 \mathrm{U}\end{array}$ \\
\hline 6 & $25 / 02 / 2009$ & Dysport $^{\circledR}$ & $\begin{array}{l}\text { Mentalis } 20 U \text { each } \\
\text { side }(40 U) \\
\text { Total: } 40 U\end{array}$ & $\begin{array}{l}\text { Mentalis } 30 \mathrm{U} \text { each side } \\
(60 U) \\
\text { Mylohyoid } 25 \mathrm{U} \text { each } \\
\text { side }(50 U) \\
\text { Masseter } 50 \mathrm{U} \text { each } \\
\text { side (100U) } \\
\text { Orbicularis oris } 3 \mathrm{U} \text { each } \\
\text { side }(6 \mathrm{U}) \\
\text { Total } 216 \mathrm{U}\end{array}$ & $\begin{array}{l}\text { Mentalis } 20 U \text { each } \\
\text { side (40U) } \\
\text { Total: } 40 U \\
\\
\end{array}$ & $\begin{array}{l}\text { Mentalis } 20 U \text { each } \\
\text { side ( } 40 U) \\
\text { Masseter } 80 U \text { each } \\
\text { side (160U) } \\
\text { Mylohyoid } 20 U \\
\text { each side (40U) } \\
\text { Total: } 240 U\end{array}$ \\
\hline 7 & $23 / 07 / 2011$ & Dysport $^{\circledR}$ & $\begin{array}{l}\text { Mentalis } 25 U \text { each } \\
\text { side ( } 50 U) \\
\text { Orbicularis oris } 2 U \\
\text { right }(2 U) \\
\text { Total: } 52 U\end{array}$ & $\begin{array}{l}\text { Mentalis } 25 \mathrm{U} \text { each side } \\
(50 U) \\
\text { Mylohyoid } 30 U \text { each } \\
\text { side }(60 U) \\
\text { Masseter } 30 U \text { each } \\
\text { side }(60 U) \\
\text { Orbicularis oris } 5 U \text { each } \\
\text { side }(10 U) \\
\text { Levator labii superioris } \\
5 U \text { each side }(10 U) \\
\text { Total } 190 U\end{array}$ & $\begin{array}{l}\text { Mentalis } 20 U \text { each } \\
\text { side (40U) } \\
\text { Total: } 40 U \\
\\
\end{array}$ & $\begin{array}{l}\text { Mentalis } 20 U \text { each } \\
\text { side ( } 40 U) \\
\text { Masseter } 60 U \text { each } \\
\text { side (120U) } \\
\text { Mylohyoid } 20 U \\
\text { each side (40U) } \\
\text { Total: } 200 U\end{array}$ \\
\hline 8 & $27 / 01 / 2012$ & Dysport $^{\circledR}$ & $\begin{array}{l}\text { Mentalis } 25 U \text { each } \\
\text { side ( } 50 U) \\
\text { Orbicularis oris } 4 U \\
\text { each side }(8 U) \\
\text { Total: } 58 U\end{array}$ & $\begin{array}{l}\text { Mentalis } 25 \mathrm{U} \text { each side } \\
(50 \mathrm{U}) \\
\text { Mylohyoid } 30 \mathrm{U} \text { each } \\
\text { side }(60 U) \\
\text { Masseter } 20 \mathrm{U} \text { each } \\
\text { side }(40 U) \\
\text { Orbicularis oris } 6 \mathrm{U} \text { each } \\
\text { side }(12 \mathrm{U}) \\
\text { Levator labii superioris } \\
6 \mathrm{U} \text { each side }(12 \mathrm{U}) \\
\text { Total } 174 \mathrm{U}\end{array}$ & $\begin{array}{l}\text { Mentalis } 20 U \text { each } \\
\text { side (40U) } \\
\text { Total: } 40 U \\
\end{array}$ & $\begin{array}{l}\text { Mentalis } 20 U \text { each } \\
\text { side ( } 40 U) \\
\text { Mylohyoid } 20 U \\
\text { each side }(40 U) \\
\text { Orbicularis oris } 6 U \\
\text { each side (12U) } \\
\text { Total: } 92 U\end{array}$ \\
\hline 9 & $24 / 08 / 2012$ & Dysport $^{\circledR}$ & $\begin{array}{l}\text { Mentalis } 20 U \text { each } \\
\text { side ( } 40 U) \\
\text { Orbicularis oris } 5 U \\
\text { each side (10U) } \\
\text { Depressor anguli } \\
\text { oris } 5 U \text { each side } \\
\text { (10U) } \\
\text { Total: } 60 U\end{array}$ & $\begin{array}{l}\text { Mentalis } 25 \mathrm{U} \text { each side } \\
(50 U) \\
\text { Mylohyoid } 30 \mathrm{U} \text { each } \\
\text { side }(60 U) \\
\text { Masseter } 20 \mathrm{U} \text { each } \\
\text { side ( } 40 U) \\
\text { Orbicularis oris } 6 \mathrm{U} \text { each } \\
\text { side (12U) } \\
\text { Total } 162 \mathrm{U}\end{array}$ & $\begin{array}{l}\text { Was not injected } \\
\text { (pregnancy) }\end{array}$ & $\begin{array}{l}\text { Mentalis } 20 U \text { each } \\
\text { side }(40 U) \\
\text { Masseter } 60 U \text { each } \\
\text { side }(120 U) \\
\text { Orbicularis oris } 5 U \\
\text { each side (10U) } \\
\text { Total: } 170 U\end{array}$ \\
\hline 10 & $14 / 06 / 2013$ & Botox $^{\circledR}$ & $\begin{array}{l}\text { Mentalis } 15 U \text { each } \\
\text { side (30U) } \\
\text { Orbicularis oris } 3 U \\
\text { each side }(6 U) \\
\text { Depressor anguli } \\
\text { oris } 3 U \text { each side } \\
\text { (6U) } \\
\text { Total: } 42 U\end{array}$ & $\begin{array}{l}\text { Mentalis } 15 \mathrm{U} \text { each side } \\
(30 \mathrm{U}) \\
\text { Mylohyoid } 15 \mathrm{U} \text { each } \\
\text { side ( } 30 \mathrm{U}) \\
\text { Orbicularis oris } 3 \mathrm{U} \text { each } \\
\text { side }(6 \mathrm{U}) \\
\text { Levator labii superioris } \\
5 \mathrm{U} \text { each side (10U) } \\
\text { Total } 76 U\end{array}$ & $\begin{array}{l}\text { Mentalis } 10 U \text { each } \\
\text { side }(20 U) \\
\text { Total: } 20 U \\
\text { ( } 9^{\text {th }} \text { injection for } \\
\text { this patient) }\end{array}$ & $\begin{array}{l}\text { Mentalis } 10 U \text { each } \\
\text { side ( } 20 U) \\
\text { Masseter } 15 \mathrm{U} \text { each } \\
\text { side ( } 30 U) \\
\text { Orbicularis oris } 3 U \\
\text { each side }(6 U) \\
\text { Total: } 56 U\end{array}$ \\
\hline
\end{tabular}




\begin{tabular}{|c|c|c|c|c|c|c|}
\hline 11 & $31 / 01 / 2014$ & Botox $^{\circledR}$ & $\begin{array}{l}\text { Mentalis } 15 U \text { each } \\
\text { side (30U) } \\
\text { Orbicularis oris } 3 U \\
\text { each side }(6 U) \\
\text { Depressor anguli } \\
\text { oris } 3 U \text { each side } \\
(6 U) \\
\text { Total: } 42 U\end{array}$ & $\begin{array}{l}\text { Mentalis } 15 \mathrm{U} \text { each side } \\
\text { (30U) } \\
\text { Mylohyoid } 15 \mathrm{U} \text { each } \\
\text { side }(30 \mathrm{U}) \\
\text { Orbicularis oris } 3 \mathrm{U} \text { each } \\
\text { side }(6 \mathrm{U}) \\
\text { Levator labii superioris } \\
5 \mathrm{U} \text { each side }(10 \mathrm{U}) \\
\text { Total } 76 \mathrm{U}\end{array}$ & $\begin{array}{l}\text { Mentalis } 10 U \text { each } \\
\text { side ( } 20 U) \\
\text { Total: } 20 U \\
\text { (10 } 10^{\text {th }} \text { injection for } \\
\text { this patient) }\end{array}$ & $\begin{array}{l}\text { Mentalis } 10 U \text { each } \\
\text { side ( } 20 U) \\
\text { Masseter } 15 U \text { each } \\
\text { side ( } 30 U) \\
\text { Orbicularis oris } 3 U \\
\text { each side }(6 U) \\
\text { Total: } 56 U\end{array}$ \\
\hline 12 & $14 / 11 / 2014$ & Botox $^{\circledR}$ & $\begin{array}{l}\text { Mentalis } 15 U \text { each } \\
\text { side (30U) } \\
\text { Total: } 30 U\end{array}$ & $\begin{array}{l}\text { Mentalis } 15 \mathrm{U} \text { each side } \\
(30 U) \\
\text { Mylohyoid } 15 \mathrm{U} \text { each } \\
\text { side }(30 U) \\
\text { Orbicularis oris } 3 \mathrm{U} \text { each } \\
\text { side }(6 \mathrm{U}) \\
\text { Levator labii superioris } \\
5 \mathrm{U} \text { each side (10U) } \\
\text { Total } 76 \mathrm{U}\end{array}$ & $\begin{array}{l}\text { Mentalis } 15 U \text { each } \\
\text { side ( } 30 U) \\
\text { Total: } 30 U \\
\text { (11 } 11^{\text {th }} \text { injection for } \\
\text { this patient) }\end{array}$ & $\begin{array}{l}\text { Mentalis } 15 U \text { each } \\
\text { side ( } 30 U) \\
\text { Orbicularis oris } 3 U \\
\text { each side }(6 U) \\
\text { Total: } 36 U\end{array}$ \\
\hline 13 & $20 / 03 / 2015$ & Botox $^{\circledR}$ & $\begin{array}{l}\text { Mentalis } 20 U \text { each } \\
\text { side (40U) } \\
\text { Mylohyoid } 5 U \text { each } \\
\text { side (10U) } \\
\text { Depressor anguli } \\
\text { oris } 3 U \text { each side } \\
(6 U) \\
\text { Total: } 56 U\end{array}$ & $\begin{array}{l}\text { Mentalis } 20 U \text { each side } \\
(40 U) \\
\text { Mylohyoid } 10 U \text { each } \\
\text { side }(20 U) \\
\text { Orbicularis oris } 4 U \text { each } \\
\text { side }(8 U) \\
\text { Levator labii superioris } \\
3 U \text { each side (6U) } \\
\text { Total } 74 U\end{array}$ & $\begin{array}{l}\text { Mentalis } 15 \mathrm{U} \text { each } \\
\text { side (30U) } \\
\text { Total: } 30 U \\
\left(12^{\text {th }} \text { injection for }\right. \\
\text { this patient) }\end{array}$ & $\begin{array}{l}\text { Mentalis } 15 U \text { each } \\
\text { side }(30 U) \\
\text { Total: } 30 U\end{array}$ \\
\hline 14 & $13 / 11 / 2015$ & Botox $^{\circledR}$ & $\begin{array}{l}\text { Mentalis } 20 U \text { each } \\
\text { side ( } 40 U) \\
\text { Mylohyoid } 5 U \text { each } \\
\text { side (10U) } \\
\text { Depressor anguli } \\
\text { oris } 3 U \text { each side } \\
(6 U) \\
\text { Total: } 56 U\end{array}$ & $\begin{array}{l}\text { Mentalis } 20 U \text { each side } \\
(40 U) \\
\text { Mylohyoid } 10 U \text { each } \\
\text { side ( } 20 U) \\
\text { Orbicularis oris } 4 U \text { each } \\
\text { side }(8 U) \\
\text { Levator labii superioris } \\
3 U \text { each side (6U) } \\
\text { Total } 74 U\end{array}$ & $\begin{array}{l}\text { Mentalis } 15 U \text { each } \\
\text { side ( } 30 U) \\
\text { Total: } 30 U \\
\text { ( } 13^{\text {th }} \text { injection for } \\
\text { this patient })\end{array}$ & $\begin{array}{l}\text { Mentalis } 15 \mathrm{U} \text { each } \\
\text { side (30U) } \\
\text { Total: } 30 \mathrm{U}\end{array}$ \\
\hline
\end{tabular}

levator labii superioris muscle ( 5 units each) and masseter muscles (15 units each). The patients did not report any specific differences between the different botulinum toxin formulations.

All four patients who received botulinum toxin treatment reported onset of effect 2 days after the first injection. There was suppression of involuntary movements of the mentalis muscles lasting 2-3 months. Even though chin trembling started to return within 2 months, the frequency of involuntary movements was usually less severe and patients could tolerate this until their next injection at 5-9 months. Treatment-related adverse effects were mouth biting and weakness when chewing; these did not affect patients' daily lives and resolved within a few weeks. No serious side effects were reported.

All four patients have continued treatment with botulinum toxin because it has improved their quality of life. Improvements were particularly evident when additional muscles (other than the mentalis) were injected, especially in patients IV-1 and IV-4 after injection of the masseter muscles. Despite the fact that no involuntary activity of masseter muscles was found in EMG testing, and that there was no specific reported association between geniospasm and masseter hypertonia, patients showed considerably better sleep quality after masseter muscles injections, which could be observed since the beginning of the injections until the end of the study. Furthermore, before treatment, patient IV-4 had reported an impact of symptoms in a social context; after treatment, his family members reported a significant improvement in his social functioning.

\section{Genomic analysis plan}

In 1997 Jarman, et al. of the University of London, published a study in which they reported two unrelated families with this same condition. One family contained 16 affected individuals over four generations. Another family contained 20 affected individuals over four generations. The authors found a mutation linked to a chromosome 9q13-q21 locus in one of the families, however this mutation was not observed in the other family [8], neither has been identified consistently in other reports [21], thus showing the genetic heterogeneity of this disease.

Few families in the world were reported with geniospasm to date and no description of Brazilian families was found. Despite the evolution of Genetics, there is still little knowledge about the genetic mechanism of this disease. We have therefore designed a clinical trial to evaluate genetic mutations amongst the five members of this Brazilian family. We plan to perform 
genomic analysis using sequencing of the exome, the fraction of the genome that encodes genes, of at least three individuals of this family (one of each generation). Exome sequencing is a modern technique that analyses exons (DNA encoding the amino acids of each gene) and provides efficient analysis of genetically heterogeneous diseases. Exome analysis allows DNA sequencing to be performed. The information is processed and organized using advanced technology, which is able to recognize, identify and note the differences between the exome studied and reference exams, and allows the identification of thousands of genetic variations [32,33]. It is especially effective in the study of rare Mendelian diseases as it identifies the genetic variants in all genes of a subject. As in hereditary geniospasm, these diseases are most often caused by rare genetic variants that are only present in a very small number of individuals.

\section{Discussion}

This is the first report of familial geniospasm in Brazil. We have traced its genealogy in a five generation family, describing its clinical features, EMG findings and treatment with botulinum toxin injections in four living members from three generations. The findings are consistent with all published pedigrees, which suggest that geniospasm is a hereditary condition, with dominant inheritance and almost complete penetrance [13,5,6,8-30]. Our observations also confirm previous findings that geniospasm occurs in infancy or early life, and that there is a relationship between the occurrence of symptoms and emotional stimuli $[3,5,7,8,12,34]$. As in previous reports $[4,5,8]$, none of our patients had any neurological impairment.

Hereditary geniospasm is characterized by involuntary movement of the mentalis muscles and, very occasionally, other facial muscles. It must be differentiated from other conditions, such as tic, facial myokymia and jaw tremor. Tic is essentially a psychical process in which a coordinated muscular movement is repeated intermittently for no useful purpose. It never occurs during sleep and each paroxysm is usually preceded by an impulse and followed by a certain feeling of satisfaction [2]. Facial myokymia can have some similarities with geniospasm considering EMG patterns [3]. However, it may occur in association with other neurological conditions, such as brain-stem tumors and multiple sclerosis. It usually affects more than one muscle and is confined to one side of the face [3]. Jaw tremor, which is considered as a spectrum of essential tremor, on the other hand, has a completely different muscular pattern (masseter activity). The tremors more often occur when the mouth is open, during speech for example, rather than when closed. It is also often associated with postural tremor, kinetic tremor of the limbs, head and voice tremors [35].

In this Brazilian family, all five members affected by geniospasm had involuntary activity of mentalis muscles, while three members had involuntary activity in the orbicularis oris, mylohyoid and levator labii superioris muscles. As we have shown in this report, EMG analysis is important for detecting muscles, other than the mentalis, that are affected. It can also be used to guide which muscles should be treated with botulinum toxin injections. Although geniospasm is a benign condition, chin trembling can be distressing and embarrassing, especially when it is first encountered [14]. However, as described in our patients, it can be treated effectively using low doses of botulinum toxin. Adverse effects were mild and uncommon, resolving within a few days without compromising patients' daily routine. Improvements in secondary bruxism symptoms were also observed following injection of the masseter muscles, including improvements in sleep quality, despite no observation of involuntary activity in EMG testing of these muscles. Furthermore, an improvement in social functioning was reported after treatment for geniospasm; this has also been reported in another case series [9].

Geniospasm is a genetically heterogeneous disease, which has been linked to a chromosome 9q13-q21 locus before [8], but this linkage has not been identified consistently in other reports [21]. Given the limited knowledge on the genetic mechanisms of this rare disease, as well as on other focal dystonias, we have designed a further clinical trial to evaluate genetic mutations through genomic analysis, using sequencing of the exome, amongst the members of this Brazilian family [32,33]. Exome analysis results of the affected individuals may help to elucidate the genetic etiology of geniospasm, which might be useful for exploring other focal dystonias as well.

\section{Conclusion}

This first report of geniospasm in a Brazilian family confirms and extends findings from previous reports of this rare genetic condition. It also shows that with good clinical evaluation using EMG, hereditary geniospasm can be easily identified and managed. Many articles describe geniospasm as a benign condition that does not impair patients' everyday life; however, members of this Brazilian family reported uncomfortable symptoms and social impairment. All reported substantial benefits during EMG-guided treatment with botulinum toxin injections over a period of 5-9 months. Even though chin trembling started to return in 2-3 months after injection, the patients reported considerably less frequency of involuntary activity, better sleep quality and improvement in social functioning until the next injection. Further studies are needed to support the autosomal-dominant inheritance of this disorder and to clarify its cause and associated factors. We therefore, have planned a follow-up clinical trial in the surviving members of this Brazilian family to detect genetic mutations associated with geniospasm using exome sequencing. The results can help to elucidate the genetic etiology of geniospasm, and the methodology may be useful for exploring other focal dystonias. 


\section{Acknowledgement}

Medical writing support was provided by Water meadow Medical, an Ash field company, part of UDG Healthcare plc (funded by Ipsen) for developing the first draft of the manuscript only. Development of the final draft and submission of the manuscript was conducted in total independence by the authors. Throughout development, the content was directed solely by the authors.

\section{Authors Roles}

Lilian Braighi Carvalho: Case series study concept and design, manuscript writing, review and critique.

Danielle Bianchini Rampim: Case series study concept and design, manuscript writing, review and critique.

Tae Mo Chung: Case series study concept and design, electromyography testing, manuscript writing, review and critique.

\section{Disclosures}

Funding Sources and Conflict of Interest. No specific funding was received for this work. The authors declare that there are no conflicts of interest relevant to this work.

\section{Ethical Statement}

The authors obtained verbal permission from the patients involved in the study.

\section{Compliance with Journal Ethical Publication Guidelines Statement}

The authors confirm that we have read the Journal's position on issues involved in ethical publication and affirm that this work is consistent with those guidelines.

\section{References}

1. Massaro D (1894) Ventisei casi de genoispasmo attraverso cinque generazioni: contributo clinico allo studio dell"ereditá fisiopatologica. I Pissani (Palermo) 1: 47-56.

2. Stocks $P$ (1923) Facial spasm inherited through four generations. Biometrika 14: 311-315.

3. Danek A (1993) Geniospasm: Hereditary chin trembling. Mov Disord 8: 335-338.

4. Mahmoudi M, Kothare SV (2014) Tongue biting: A case of sporadic geniospasm during sleep. J Clin Sleep Med 10: 1339-1340.

5. Destee A, Cassim F, Defebvre L, J Guieu (1997) Hereditary chin trembling or hereditary chin myoclonus? J Neurol Neurosurg Psychiatry 63: 804-807.

6. Wadlington WB (1958) Familial Trembling of the chin. J Pediatr 53: 316-321.

7. Jain P, Sharma S, Aneja S (2015) Hereditary chin-trembling. Indian Pediatrics 52: 720.

8. Jarman PR, Wood NW, Davis MT, Davis PV, Bhatia KP, et al. (1997) Hereditary geniospasm: linkage to chromosome 9q13-q21 and evidence for genetic heterogeneity. Am J Hum Genet 61: 928-933.
9. Gordon K, Cadera W, Hinton G (1993) Successful Treatment of Hereditary Trembling Chin With Botulinum Toxin. J Child Neurol 8: 154-156.

10. Chalaupka FD, Bartholini F, Mandich G, Mandich G, Turro M (2006) Two new families with hereditary essential chin myoclonus: clinical features, neurophysiological findings and treatment. Neurol Sci 27: 97-103.

11. Bakar M, Zarifoglu M, Bora I, Turan F, Sen C, et al. (1998) Treatment of hereditary trembling chin with botulinum toxin. Mov Disord 13: 845-846.

12. Ganner-Innsbruck H (1938) Inherited trembling chin in a Tyrolean community. Zeitschrift Fur Die Gesamte Neurologie Und 161: 259-266.

13. Grossman BJ (1957) Trembling of the Chin - An Inheritable Dominant Character. Pediatrics 19: 453.

14. Laurance BM, Matthews WB, Diggle JH (1968) Hereditary Quivering of the Chin. Arch Dis Child 43: 249-251.

15. Johnson LF, Kinsbournet M, Renuartt AW (1971) Hereditary Chin-trembling with Nocturnal Myoclonus and Tongue-biting in Dizygous Twins. Dev Med Child Neurol 13: 726-729.

16. Becker $H$ (1982) Hereditary quivering of the chin in a West Spanish family. Arch Psychiatr Nervenkr 231: 509-518.

17. Blaw ME, Leroy RF, Steinberg JB, Herman J (1989) Hereditary Quivering Chin and Rem Behavioral-Disorder. Ann Neurol 26: 471.

18. Danek A, Gams M, Garner C (1991) Trembling Chin (Geniospasm). Aktuelle Neurol 18: 124-127.

19. Alsager DE, Bowen $P$, Bamforth JS (1991) Trembling Chin - A Report of this Inheritable Dominant Character in A 4-Generation Canadian Family. Clin Genet 40: 186-189.

20. Soland VL, Bhatia KP, Sheean GL, Marsden CD (1996) Hereditary Geniospasm: Two New Families. Movment Disorders 11: 744-746.

21. Grimes DA, Han F, Bulman D, Nicolson ML, Suchowersky $\mathrm{O}$ (2002) Hereditary Chin Trembling: a new family with exclusion of the chromosome 9q13-q21 Locus. Mov Disord 17: $1390-1392$.

22. Papapetropoulos S, Singer C (2007) Sporadic Geniospasm (Chin Trembling): Report of a Case. Movement Disorders 22: 434-443.

23. Erer S, Jankovic J (2007) Hereditary chin tremor in Parkinson's disease. Clin Neurol Neurosurg 109: 784-785.

24. Kharraz B, Reilich P, Noachtar S, Danek A (2008) An episode of geniospasm in sleep: toward new insights in to Pathophysiology? Mov Disord 23: 274-276.

25. Aggarwal A, Warren JE, Warren JD, Thompson PD (2009) Facial Reflex Hyperexcitability in Geniospasm Suggests a Brainstem Origin. Movememt Disorders 24: 783-784.

26. Takashim H, Ohde M, Baba K (1973) Pedigree of Hereditary trembling of the chin. Jpn J Hum Genet 18: 88-89.

27. Goraya JS, Virdi V, Parmar V (2006) Recurrent Nocturnal Tongue Biting in a Child with Hereditary Chin Trembling. J Child Neurol 21: 985-987.

28. Türkmen Ç, Özen B, Ertaşoğlu H, Ağan K, İnce Günal D (2008) Hereditary geniospasm in a Turkish Family. Parkinson Hast Hareket Boz Der 11: 17-19.

29. Diaz S, Maria Clara S, Federico M (1999) Hereditary Chin Tremor/Myoclonus: a report from Latin America. Mov Disord 14: 180-182. 
30. Torres L, Velez M, Cosentino C (2001) Chin tremor in a Peruvian family. Rev Neurol 33: 958-959.

31. Van Gerpen JA, Matsumoto JY, Ahlskog JE, Maraganore DM, McManis PG (2000) Utility of an EMG mapping study in treating cervical dystonia. Muscle Nerve 23: 1752-1756.

32. Ng SB, Turner EH, Robertson PD, Flygare SD, Bigham AW, et al. (2009) Targeted capture and massively parallel sequencing of 12 human exomes. Nature 461: 272-276.
33. Ng SB, Buckingham KJ, Lee C, Bigham AW, Tabor HK, et al. (2010) Exome sequencing identifies the cause of a mendelian disorder. Nat Genet 42: 30-35.

34. Frey E (1957) Ein streng dominant erbliches Kinnmuskelzittern. In: Grossman BJ, Trembling of the Chin - An Inheritable Dominant Character. Pediatrics 19: 453.

35. Hernandez NC, Louis ED (2015) Jaw tremor resulting in broken teeth: on the essential tremor spectrum. Tremor Other Hyperkinet Mov 5: 354. 